\title{
Investigating the Effect of Relative Width on Momentum Transfer between Main Channel and Floodplain in Rough Rectangular Compound Channel Sunder Varius Relative Depth Condition
}

\author{
Shima Bahadori' ${ }^{1}$ Mehdi Behdarvandi Askar ${ }^{2 *}$ \\ ${ }^{1}$ Master's Student, Department of Offshore Structures, Faculty of Marine Engineering, \\ Khorramshahr University of Marine Science and Technology, Khorramshahr, Iran \\ ${ }^{2}$ Assistant Professor, Department of Offshore Structures, Faculty of Marine Engineering, \\ Khorramshahr University of Marine Science and Technology, Khorramshahr, Iran \\ Email:Shb.8564@yahoo.com, *sazehenteghal@yahoo.com
}

Received 11 January 2016; accepted 10 April 2016; published 13 April 2016

Copyright (C) 2016 by authors and Scientific Research Publishing Inc.

This work is licensed under the Creative Commons Attribution International License (CC BY). http://creativecommons.org/licenses/by/4.0/

(c) (1) Open Access

\begin{abstract}
Compound section is referred to a section the surface of which is made of several sub-sections with different flow characteristics. The difference in the hydraulic and geometry characteristics causes a complexity in flow hydraulic and creates an interaction between the main channel and floodplains, resulting in an apparent shear stress and a transverse momentum transfer. The amount of such a stress plays an important role in many river engineering measures [1]. Due to the flow complexity, the common approximate analytical methods are not enough to identify the flow profile. The FLOW3D Software with its great features in three-dimensional analysis of flow field is used as a tool to investigate the shear stress in a direct symmetrical compound rectangular channel. After the simulation of models, it is found that an increase in the relative width and relative depth parameters decreases the percentage of apparent shear stress and an increase in the relative roughness causes it to be increased [2].
\end{abstract}

\section{Keywords}

Compound Channel, Momentum Transfer, Relative Roughness, Relative Depth, Relative Width

${ }^{*}$ Corresponding author.

How to cite this paper: Bahadori, S. and Askar, M.B. (2016) Investigating the Effect of Relative Width on Momentum Transfer between Main Channel and Floodplain in Rough Rectangular Compound Channel Sunder Varius Relative Depth Condition. Open Journal of Geology, 6, 225-231. http://dx.doi.org/10.4236/ojg.2016.64020 


\section{Introduction}

Compound section is referred to a section the surface of which is made of several sub-sections with different flow characteristics. Each section consists of a main creek or channel and two floodplains. A natural river, from which a flow passes after supercharging of the waterway and entering into the adjacent lands at the time of flooding, is an example of a compound section [1].

In the absence of a flood, the flow only transits from the main waterway and floodplains generally remain without any flow, resulting in a lesser degree of roughness of the main channel compared to the floodplains. This difference in roughness during floods and flowing of stream from flood plains leads to a difference between the velocities in the sub sections. In addition to the difference in roughness and velocity between sub-sections of the compound channel, other geometry and hydraulic parameters affect hydraulic of the flow in the compound channels. Relative depth, relative width, transverse slope of floodplains, floor slope, side slope of walls as well as figure ratio are among effective parameters. The difference in values of the aforementioned parameters is followed by the complexity of the hydraulic flow. Such differences cause an interaction between deep flow in the main channel and shallow flow in the floodplains the result of which is a shear stress which is in addition to the bed and walls shear stress and is generally called apparent shear stress. This apparent shear stress dramatically violates flow pattern and boundary shear stress [3].

Formation of apparent shear stress and vortices causes the flow to be slow in the main channel which indicates the transition of momentum in the width of the channel [1].

As a result of this complexity in the hydraulic of the flow, the common approximate analytical methods are ineffective to be used to obtain flow specifications and equations in compound channels.

In most river engineering measures to anticipate the transmission capacity, velocity and boundary shear stress distributions caused by the floods are needed. Apparent shear stress and transverse momentum transfer resulted from it play important roles in the calculation of flow rate and secondary flows to identify and control the sediment transport mechanism in order for protecting coasts, organizing rivers, designing a stable channel, analyzing scouring as well as transmitting the contamination [4].

A wide range of studies have been conducted in connection with symmetrical compound rectangular channels and the investigation of the apparent shear stress in them as well as the effect of various parameters on them [5].

Zhelzinkov (1971), after conducting several studies on compound channels, for the first time, introduced the ratio of depth of the main channel to the depth of the floodplain as a major factor in the hydraulic of compound channels which was later called effective depth. Myers (1978), using a Preston tube, measured the shear stress distribution around a compound channel including a deep section and a floodplain in a flume with the size of $9.15 \mathrm{~m}$. Using the obtained results, he quantitized the transition of momentum and stated the dangers caused by ignoring this phenomenon in the analysis of the compound channel. Myers called the momentum transfer phenomenon between the main channel and the floodplain "kinetic effect". By considering the ratio of the depth of the floodplain to the depth of the whole channel as the relative depth, he studied force changes, apparent shear stress and the intensity of vortices using the Reynolds number and the relative depth. He concluded that by increasing the relative depth and the Reynolds number, the apparent shear stress is reduced and the maximums value of apparent shear force can reach $25 \%$ of the component of the main channel water weight [6].

Knight and Demetriou (1983) proposed some equations to determine the transverse momentum transfer based on experimental data and found that for compound channels with low relative depth and high floodplain width, apparent shear force increases on the floodplain boundary wall/main channel [2]. Knight (1984) studied the effect of roughness difference between the floodplain and main channel on the transverse momentum transfer by the gradual roughening of floodplains in 6 steps in a symmetrical compound channel. He also investigated the effect of such a decrease on boundary shear and flow forces in the roughest state of the floodplain by reducing the width of the floodplain. He considered 4 dimensionless parameters that contained the ratio of the floodplain width to the main channel width, the ratio of floodplain depth to the depth of the main channel, the ratio of floodplain roughness to the main channel roughness and the ratio of the main channel width to the main channel depth. The experiments were conducted in a channel with 15 meters length and 6.1 meters width. After the measurement of shear velocity and force on a humid environment, he proposed some equations to calculate the apparent shear stress in three states of horizontal, vertical and oblique apparent connection faces and came to the conclusion that the percentage of total shear force of floodplains significantly increases with increasing the roughness of floodplains and for a smooth channel (the roughness ratio of the 
floodplain to the main channel is equal to 1 ), which is the geometry for the constant main channel (width to depth ratio is equal to 1 ), the percentage of the shear force of floodplains for a known width ratio increases with increasing of the depth ratio [7].

Shinio and Knight (1991) conducted a series of experiments in a stable creek belonging to the University of Birmingham with the length of $56 \mathrm{~m}$ and the width of $10 \mathrm{~m}$ with the capability of variability in the width of floodplains with a compound trapezoidal cross section in the main creek and floodplains and achieved remarkable results. Isam Al-Khatib (1999) examined the influence of the cross section geometric parameters, especially the main channel width and the main channel wall height on the shear stress distribution changes in the floodplain and main channel of a compound rectangular channel [8]. The flume under the experiment was 9 meters long, 0.67 meters wide and 0.75 meters deep and the shear stress was measured using a Preston tube. The shear stress pattern was obtained for 11 different depths. Al-Khatib came to the conclusion that by increasing the width and height of the wall of the main channel, its average bed shear stress also increased. Moreover, he found that wider floodplains due to loss of energy to floodplains and greater momentum transfer than floodplains with narrower width will cause a reduction in the main channel shear. With regard to the relationship of average shear stress ratio in the main channel bed to average shear stress in floodplain bed with relative depth (depth of floodplain to the main channel ratio) Al-Khatib concluded that for low relative depth values, the shear stress ratio is greater than 1 and for greater relative depths, the ratio is close to or slightly smaller than 1 . He stated the reason to be the greater momentum transfer in the small relative depth and lower momentum transfer in greater relative depths. Fazli and Rezai (2010) by keeping constant the geometric dimensions of the main channel and changing the width of floodplains and the relative depth investigated the effect of these parameters on shear stress in a compound channel. Preston tube was used to directly measure the shear stress and it was calculated using the exsiting hydraulic relations. The flume under the experiment had a length of 18 meters, width of $1200 \mathrm{~mm}$, height of $400 \mathrm{~mm}$ and the slope of 0.0023 and 4 values were considered for the floodplain width. Fazli and Rezaei came to the conclusion that by increasing the width of floodplains, the degree of shear stress is reduced. Regarding the effect of the relative depth on the shear stress they came to the conclusion that by increasing the relative depth, the shear stress increases and by increasing the relative depth more than 0.4 , the impact of an increase in the floodplain width to an increase in the shear stress can be ignored [9].

Since no study had directly investigated the effect of some parameters as well as the apparent shear stress with the FLOW3D software, this study aims to investigate the effects of relative depth, the relative width, and relative roughness on the transfer of momentum and apparent shear stress in a rectangular, straight, and symmetric compound channel with the use of the FLOW3D software [10].

\section{Materials and Methods}

In order to investigate the effect of geometric and hydraulic parameters of the compound channel on the apparent shear stress, these features, using dimensional analysis, were divided into three dimensionless parameters including relative roughness, relative depth, and relative width (Table 1)

$$
D_{r}=\frac{H-h}{H}
$$

is the relative depth parameter which is equal to the ratio of floodplain depth to the main channel depth.

$$
n_{r}=\frac{n_{f p}}{n_{m c}} \text { is the relative roughness parameter which is equal to the ratio of floodplain roughness to the main }
$$

channel roughness.

\begin{tabular}{cc} 
Table 1. Tested parameters. & \\
\hline Tested Parameters & Tested Parameters Value \\
\hline Relative Roughness & $1,2,3,5$ \\
$\mathrm{D}_{50}$ Main Channel $(\mathrm{mm})$ & $1.31,4.6,10$ \\
Relative Width & $1.25,2,2.5,5$ \\
Relative Depth & $0.1,0.35,0.5$ \\
\hline
\end{tabular}


$W_{r}=\frac{\frac{b_{m c}}{2}+b_{f p}}{\frac{b_{m c}}{2}}$ is the relative width parameter,

where, in the above parameters:

$H$ : The main channel depth

$h$ : The floodplain depth

$n_{f p}$ : Size of the floodplain roughness

$n_{m c}$ : The main channel roughness

$b_{f}$ : The floodplain width

$b_{c}$ : The main channel width

Physical properties of the simulated channel is a rectangular, straight, and symmetric compound channel including a main channel and two floodplains with the main channel width of $0.5 \mathrm{~m}$, main channel height of $0.5 \mathrm{~m}$, total length of $12 \mathrm{~m}$, and the bottom steepness of 0.001 (Figure 1).

\section{Numerical Models}

The FLOW 3D software is a powerful hydraulic simulator application in the field of fluid dynamics with threedimensional flow field analysis. The equations governing in the model, like other similar models, are NavierStokes equations and the conservation of mass equation.

In order to model the channel in this application, we need to define the General Conditions (including the simulation of all systems), physical conditions, geometry and model-solving network, adjusting the outputs and its related options. Celsius Degrees was chosen for system units, SI, and temperature [7].

In physical terms, the software allows that, according to the principles of the physics governing the phenomenon, the relevant conditions are chosen. The physical conditions governing this study are gravity and viscosity and turbulence. The turbulence in this software is stimulated by five models and the model used in this research was Re-Normalisation Group (RNG). In this model of turbulence, constant values which were experimentally calculated in K-model are implicitly derived.

After that, the fluid should be defined. The selected fluid of this study is the 20-degree-Celsius water.

The next step is to define the geometry and resolve network, which is of importance in simulation. The FLOW3D enables the user to depict a lot of fluid phenomena with the tools available in the software. Defining the channel geometry, resolve network should be defined [11]. The defined resolve network of the software is in the form of regular (cubic) resolve network including network size, number of cells and their dimensions in three coordinates of $\mathrm{X}, \mathrm{Y}$, and $\mathrm{Z}$ and boundary conditions. The smaller the size of network cell dimensions and the greater their number, the higher the capability and precision of the program for simulation will be.

In defining the boundary conditions according to the existing conditions of the model, some properties can be assigned to every aspect of the resolve network. In this study, volume flow rate requirement and outflow boundary condition (Outflow), and wall and symmetry conditions are considered for upstream, the lower boundary, walls and floors, respectively.

After the definition, implementation and completion of the simulation model, the apparent shear stress value and its percentage can be obtained using the provided shear stress outputs and equations (1) and (2).

$$
\frac{\rho g A_{m c h} S_{f}}{2}=\int \tau_{0} \mathrm{~d} P+A S F
$$

The left side of the equation shows the amount of the shear force in half of the main channel and the ASF represents the apparent shear force and the integral terms show the shear force for the whole channel.

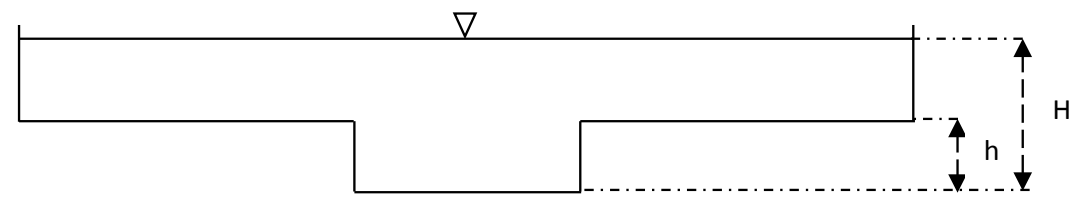

Figure 1. Schematic view of a compound channel relative depth parameter. 


$$
\% A S F=\left(\frac{2 A S F}{\rho g A_{t} S_{f}}\right) \times 100
$$

In this regard, \% ASF is the percentage of the apparent shear force or relative apparent shear force and ASF represents apparent shear force obtained from Equation (1), and the denominator indicates the total channel shear force (Figure 1).

\section{Conclusion}

In order to evaluate the effect of relative roughness parameters and the relative width on the apparent shearing stress, the following charts are drawn.

The horizontal axis is the percentage of apparent shear stress and vertical axis shows the relative roughness.

In all the above graphs, in each main channel constant roughness size, in all 3 relative depths and 4 relative widths, the percentage of apparent shear stress increases with an increase in the relative roughness size.

In the graphs drawn for the relative depth of 0.15 which is the lowest relative depth of simulation, in comparison with other two relative depths, graphs are steeper and the rate of change for the apparent shear stress is more increased. The reason for this is that in lower relative depths, the relative roughness is more apparent and has greater impact on the transfer of momentum and consequently on the apparent shear stress; however, with increasing relative depth, the effect of relative roughness is less and the changes of apparent shear stress are less (Figure 2).

For each relative roughness, the percentage of the apparent shear stress decreases by an increase in relative depth and the percentage of the apparent shear stress are reduced by increased relative depth which means increased depth in floodplains. It is because of the changes in the nature of the compound channel from a compound section to a simple rectangular channel, with increasing depth.

For a main channel roughness size and the relative depth as well as the constant relative roughness, with an increase in the relative width the percentage of the apparent shear stress is reduced. The reason for such a reduction of apparent shear stress percentage by increasing the relative width is that as floodplains become wider, the momentum transfer becomes greater and therefore this percentage decreases (Figure 2, Figure 3).

By comparison of the charts relevant to all 3 main channel roughness sizes it becomes clear that when the main channel roughness size is at its least state meaning 0.00131 meters, changes in shear stress with relative width are more noticeable than of the two other roughness sizes. So that the rate of apparent shear stress percentage change in this size of roughness is decreasing by increasing the relative width. The reason is that in a smoother main channel, the impact of the increasing width of floodplains on the percentage of the apparent shear stress due to lack of roughness, is more influenced by the relative width. However, in more rough substrates, the effect of changes in the relative width drops against roughness and the rate of changes of shear stress

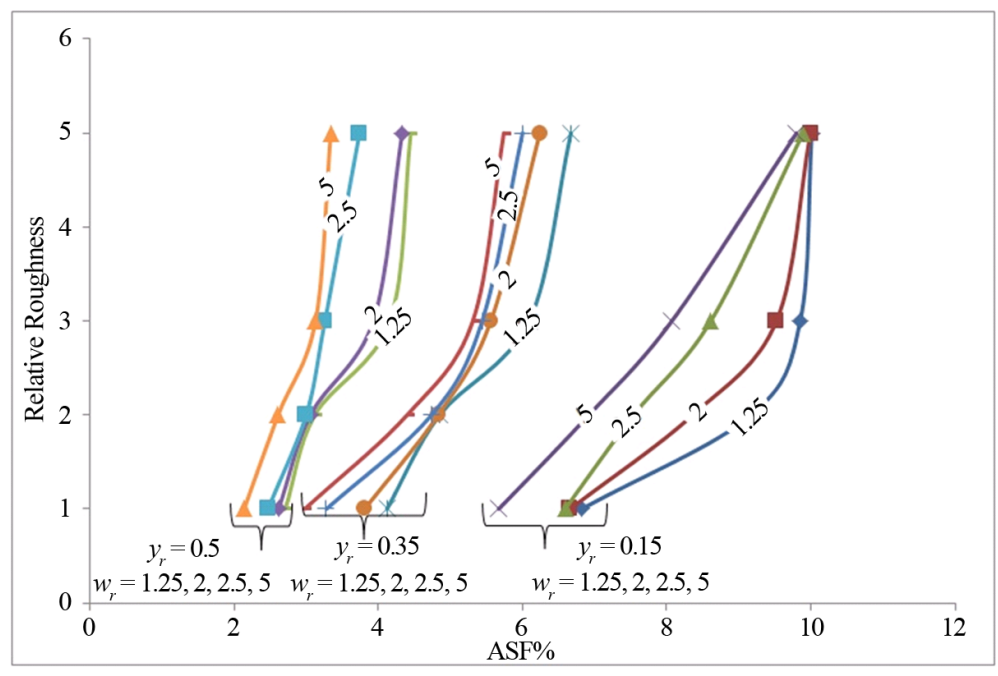

Figure 2. The main channel roughness size $0.00131 \mathrm{~m}$. 


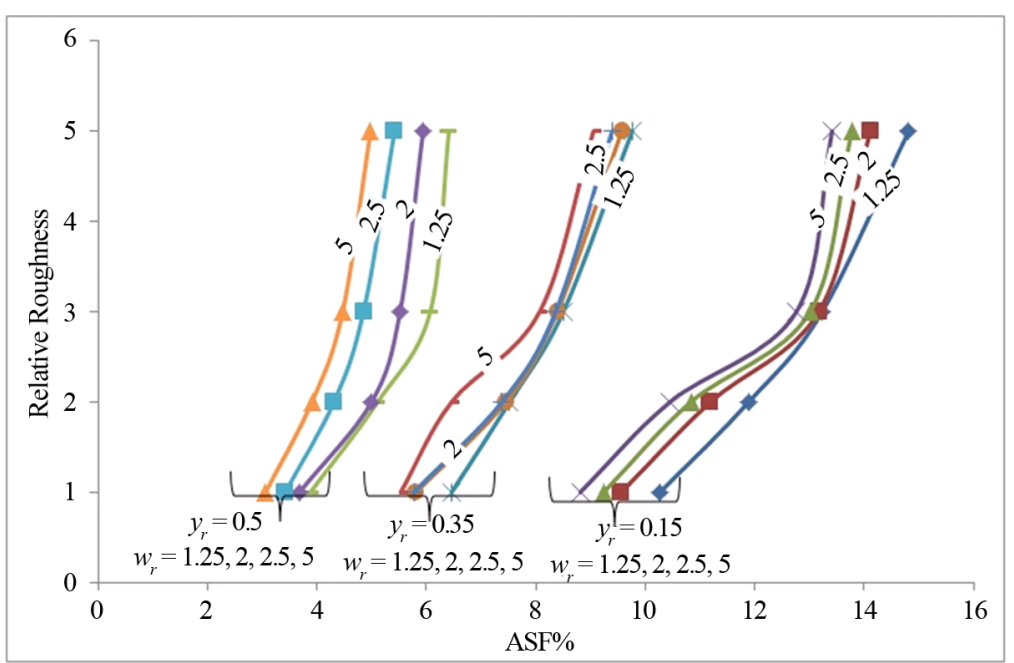

Figure 3. The main channel roughness size $0.0046 \mathrm{~m}$.

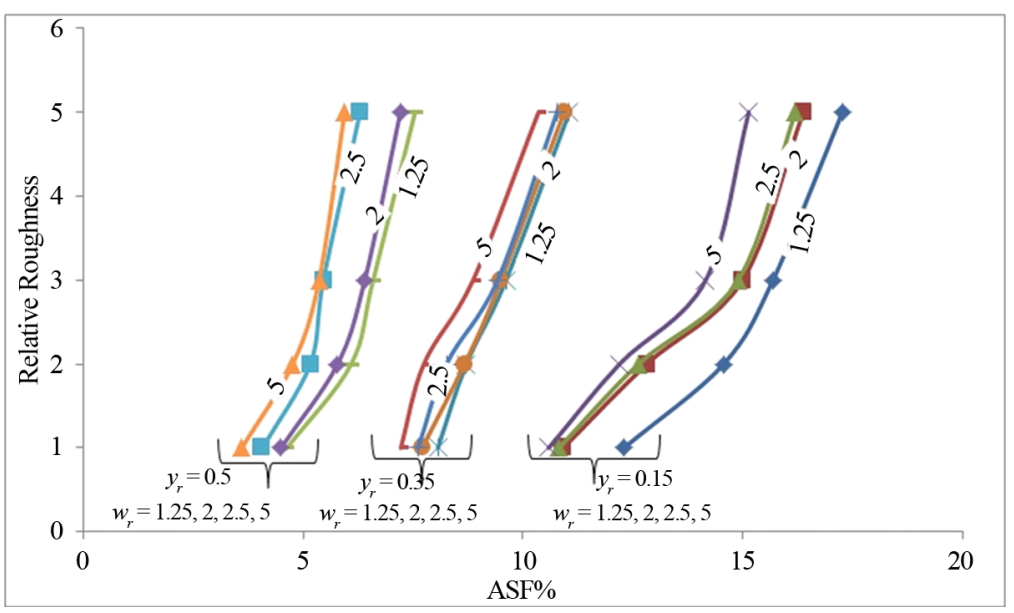

Figure 4. The main channel roughness size $0.01 \mathrm{~m}$.

decreases (Figure 4).

\section{References}

[1] Askar, B., Moghadam, M.F. and Habib, M.M.J. (2013) Comparison of Energy Methods and Momentum in the Study of Momentum Transfer among Sub-Sections of a Compound Section. Journal of Water Resources Engineering, VI, 114.

[2] Zheleznyakov, G.V. (1965) Relative Deficit of Mean Flow of Instable River Flow, Kinematic Effect in River Beds with Floodplains. Proceedings of 11th International Congress of the International Association for Hydraulic Research, Leningrad, 1965, 34-40.

[3] Nugroho, O. and Ikeda, S. (2007) Comparison Study of Flow in a Compound Channel: Experimental and Numerical Method Using Large Eddy Simulation SDS-2DH Model. ITB Journal of Engineering Science, 39B, 67-97.

[4] Myers, W.R.C. (1978) Momentum Transfer in a Compound Channel. Journal of Hydraulic Research, 16, $139-150$. http://dx.doi.org/10.1080/00221687809499626

[5] Rice, C.E. (1974) Hydraulics of Main Channel-Floodplain Flows. Research Project Technical Completion Report, Oklahoma State Univ.

[6] Ali, A. and Manouchehr, F.M. (2011) Checking the Shear Stress Changes in the Compound Rectangular Level. The 9th International Seminar on River Engineering, Shahid Chamran University, Ahvaz, 2011, 56-62.

[7] Yonesi, H., Omid, M.H. and Ayyoubzadeh, S.A. (2013) The Hydraulics of Flow in Non-Prismatic Compound Channel. 
Journal of Civil Engineering and Urbanism, 3, 342-356.

[8] Fazli, M. and Rezaei, B. (2010) Assessment of Changes in Shear Stress for the Compound Prismatic Channels Due to Euclid-Based Geometry. The 9th Conference on Iran Hydraulic, Tarbiat Modarres University, Tehran.

[9] Knight, D.W. and Hamed, M.E. (1984) Boundary Shear in Symmetrical Compound Channel. Journal of Hydraulic Engineering, 110, 1412-1430.

[10] Al-Khatib, I.A. (1996) Boundary Shear Stress in Rectangular Compound Channels. Journal of Engineering and Enviromental Science, 23, 9-18.

[11] Ching, E. (2011) Apparent Shear Stress in Symmetric Straight Compound-Channel Flow. International Journal of Environmental Protection, 1, 28-32. 